\title{
Switch in KRAS mutational status during an unusual course of disease in a patient with advanced pancreatic adenocarcinoma: implications for translational research
}

\author{
Sibylle Baechmann ${ }^{1 \dagger}$, Steffen Ormanns ${ }^{1+}$ (D) Michael Haas ${ }^{2}$, Stephan Kruger ${ }^{2}$, Anna Remold ${ }^{1,2}$, \\ Dominik Paul Modest ${ }^{2}$, Thomas Kirchner ${ }^{1,4}$, Andreas Jung ${ }^{1,4}$, Jens Werner ${ }^{3}$, Volker Heinemann ${ }^{2,4 \dagger}$ and \\ Stefan Boeck ${ }^{2^{*} \dagger}$
}

\begin{abstract}
Background: Despite the introduction of novel effective treatment regimens like gemcitabine plus nab-paclitaxel and FOLFIRINOX, pancreatic ductal adenocarcinoma (PDAC) remains one of the most aggressive epithelial tumors. Among the genetic alterations frequently found in PDAC, mutations in the KRAS gene might play a prognostic role regarding overall survival and may also have the potential to predict the efficacy of anti-EGFR treatment.
\end{abstract}

Case presentation: We report the clinical case of a 69 year old Caucasian female that was diagnosed with histologically confirmed locally advanced PDAC with lymph node involvement in August 2010. At the time of first diagnosis, tumor tissue obtained from an open regional lymph node biopsy showed a poorly differentiated adenocarcinoma with a wild type sequence within exon 2 (codon 12/13) of the KRAS gene. The patient initially received single-agent gemcitabine and a subsequent 5-FU-based chemoradiotherapy with a sequential maintenance chemotherapy with oral capecitabine resulting in a long term disease control. Local disease progression occurred in May 2014 and the patient underwent pancreaticoduodenectomy in September 2014. A novel KRAS gene mutation (c.35G > T, p.G12 V) in exon 2 (codon 12) was detected within the surgical specimen. As of January 2016 the patient is still alive and without evidence of the underlying disease.

Conclusions: Specifically in the context of clinical trials and translational research in PDAC a re-assessment of molecular biomarkers, i. e. KRAS, at defined time points (e. g. relapse, disease progression, unusual clinical course) may be indicated in order to detect a potential switch in biomarker status during the course of disease.

Keywords: Pancreatic ductal adenocarcinoma (PDAC), KRAS mutation, Tumor heterogeneity

\section{Background}

Pancreatic ductal adenocarcinoma (PDAC) is one of the most aggressive epithelial tumors worldwide. In most patients it represents a deadly disease [1] due to an advanced stage at the time of diagnosis and the difficulties in therapeutic treatment, but also due to genetic heterogeneity [2]. Surgical resection remains the only

\footnotetext{
* Correspondence: stefan.boeck@med.uni-muenchen.de

'Equal contributors

${ }^{2}$ Department of Internal Medicine III and Comprehensive Cancer Center, Klinikum Grosshadern, Ludwig-Maximilians University of Munich, Marchioninistr. 15, 81377 Munich, Germany

Full list of author information is available at the end of the article
}

curative treatment option for localized PDAC. During the last decade, systemic treatment with single-agent gemcitabine has evolved as standard chemotherapy for the adjuvant and palliative treatment setting $[3,4]$. Gemcitabine offers a median survival of about 5 to 7 months in patients with advanced disease and shows comparatively good tolerability [5]; more recently, gemcitabine-based combination regimens with the oral epidermal growth factor receptor (EGFR) inhibitor erlotinib or together with nab-paclitaxel [6] showed a statistically significant improvement in overall survival (OS). The development and progression of PDAC 
include different genetic alterations in oncogenic activation, loss of tumor-suppressor gene function and overexpression of receptor-ligand systems [7, 8]. Among these genetic alterations, mutations in the $K R A S$ gene, which often are already present in precursor lesions, play an important role in tumor development and progression [8]. Gain of function mutations in the KRAS gene are detected in about 70 to $90 \%$ of PDAC cases [9], commonly as point mutations in exon 2 (codon 12/13), most frequently as p.G12D (c.35G > A) or p.G12 V (p.35G > T). Several studies showed that constitutively activating KRAS mutations are associated with worse OS, whereas KRAS wildtype status is associated with improved OS in PDAC [7, 10, 11]. Thus, in PDAC, KRAS mutations may be regarded as prognostic biomarker. The role of KRAS mutational status as predictive biomarker regarding the use of EGFR-targeting agents like erlotinib in advanced PDAC still remains a matter of debate to date [12-14].

Here, we report the case of a PDAC patient with an unusual clinical course: the tumor of the patient harbored a wildtype KRAS gene at the time of initial PDAC diagnosis; however, upon disease progression 4 years later, a mutation within exon 2 of the $K R A S$ gene was detectable.

\section{Case presentation}

A currently 75-year-old woman was diagnosed with locally advanced PDAC at our comprehensive cancer center (CCC) in 2010. An explorative laparotomy in August 2010 showed metastatic disease spread extensively to regional lymph nodes and thus the primary tumor in the pancreatic head was not resected. By CT imaging criteria no other distant metastatic disease was evident. Lymph nodes were sampled surgically from the right gastric artery, the hepatic artery, the coeliac trunc and from the interaortocaval region; in all samples, tumor infiltration by a poorly differentiated adenocarcinoma was confirmed by histology. Immunohistochemical staining was positive for CK7, CK20 and CA 19-9 (with CDX-2 being negative). At that time point an additional analysis for KRAS mutational status and EGFR protein expression (which were conducted within a translational research project) detected a wildtype sequence of KRAS exon 2 by pyrosequencing and a moderately positive immunohistochemical staining for membranous EGFR expression in about $80 \%$ of the tumor cells.

The patient initially received systemic chemotherapy with three cycles of standard dose $\left(1000 \mathrm{mg} / \mathrm{m}^{2}\right)$ gemcitabine between September and December 2010. Imaging studies in January 2011 confirmed stable disease and the CA 19-9 levels decreased from $3700 \mathrm{U} / \mathrm{ml}$ at first diagnosis to $180 \mathrm{U} / \mathrm{ml}$. In February 2011 5-FU-based chemoradiotherapy (30 Gy) was applied at an external hospital.
During re-exploration performed in May 2011 surgical biopsies from the peritoneum histologically confirmed metastatic disease of PDAC; thus, no attempt to resect the primary tumor in the pancreas was performed. We then decided, also based on the wish of the patient, to re-start systemic chemotherapy and treatment with oral capecitabine was initiated in July 2011 and given until April 2012. During this chemotherapy, a further decline of CA 19-9 values was observed (nadir: $30 \mathrm{U} / \mathrm{ml}$ ) and repeated $\mathrm{CT}$ imaging did not show any signs of local disease progression or metastatic disease (as assessed by imaging criteria). After a treatment rest for two years (beginning in May 2012), local tumor progression of the pancreatic primary was observed within a $\mathrm{CT}$ scan in May 2014. Again, no radiographic signs of distant metastasis were observed. Systemic chemotherapy with single-agent gemcitabine was re-introduced in June 2014 resulting in a CA 19-9 decrease from $690 \mathrm{U} / \mathrm{ml}$ at disease progression to $380 \mathrm{U} / \mathrm{ml}$ after three gemcitabine applications. Due to a progressive duodenal infiltration with clinical and endoscopic signs of gastrointestinal obstruction, a surgical re-exploration was performed in September 2014. Intraoperatively, no signs of peritoneal carcinomatosis were apparent and a liver biopsy showed no signs of malignancy. Thus, the pancreatic primary was removed by a pylorus preserving pancreaticoduodenectomy (modified Whipple-Kausch procedure). The tumor was classified as ypT3 ypN0 (0/15) L0 V0 Pn0, ductal adenocarcinoma G3, R0 resection (according to UICC criteria, TNM classification 7th edition, 2010). An additionally executed KRAS mutational analysis at this time point revealed a new point mutation p.G12 V (c.35G > T) in exon 2, codon 12. After surgery, CA 19-9 values decreased to levels of $20 \mathrm{U} / \mathrm{ml}$. The patient was offered adjuvant chemotherapy with S-1 (tegafur, gimeracil, oteracil) after pancreaticoduodenectomy and started this treatment December 2014; however, S-1 was tolerated poorly due to gastrointestinal toxicity (diarrhea grade 4 and accompanying renal insufficiency) and was therefore terminated in March 2015. As of January 2016 the patient is still alive and without clear evidence of the underlying disease. An overview of this unusual disease course is shown within Fig. 1.

\section{Discussion}

Up to now, no prognostic or predictive tissue biomarker is available for PDAC [12]. In contrast to other diseases like breast, lung or colorectal cancer no specific biomarker has been validated for clinical use in pancreatic cancer and several clinical and translational trials are ongoing in order to better define the molecular basis of this disease and to search specifically for predictive markers for treatment efficacy. Thus, only limited data is available on the clinical role of biomarkers in PDAC 


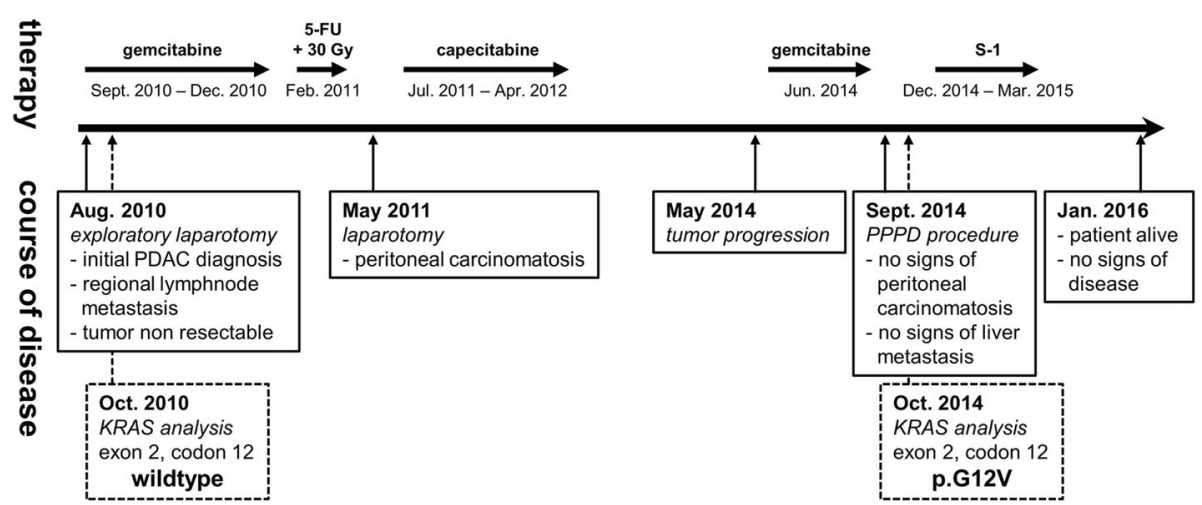

Fig. 1 Therapy, procedures and KRAS mutational status over the time course of the disease (5-FU = 5-fluouracile, Gy $=$ Gray, PPPD $=$ pylorus preserving pancreaticoduodenectomy)

[12]; specifically, there are no clear recommendations at which time points biomarkers should be assessed. In CRC for example, a good correlation between biomarker results from the primary tumor and from (metachronous) CRC metastases has been reported, resulting in the acceptance of e. g. RAS status of primary tumor tissue in patients with a metachronous relapse [15]. In contrast, in other diseases like breast cancer a switch in e. g. Her2/neu (ERBB2) status is well known resulting in the recommendation of repeated tumor biopsies at relapse or disease progression [16]. At least to our knowledge, studies investigating this issue have not yet been performed in PDAC.

Within this manuscript we report a rather unusual clinical course of a PADC patient, with a corresponding switch in KRAS mutational status during the course of disease. Of note, we detected the new KRAS mutation upon disease progression in September 2014; furthermore, it may be important to highlight the fact that this patient did not receive previous anti-EGFR treatment (e.g. with erlotinib) before the detection of the new KRAS mutation.

Several possible explanations may be hypothesized for the observation of a KRAS switch during the course of disease in our PDAC patient:

1. Appearance of a truly new tumor KRAS mutation upon disease progression in September 2014 without previous application of agents targeting the EGFR pathway:

The reason for tumor progression could be caused by an evolved new mutation event in the KRAS gene, specifically in the light of selection pressure during previous treatment with chemotherapy and radiotherapy. In colorectal cancer, increasing evidence exists that the appearance of new KRAS mutations during treatment with agents targeting the EGFR (like cetuximab or panitumumab) may be linked to an acquired resistance to anti-EGFR therapy $[17,18]$. Of note, our patient did not receive anti-EGFR treatment for example with erlotinib before the detection of the new KRAS mutation. If other treatments like cytotoxic chemotherapy (gemcitabine, fluoropyrimidines) or radiotherapy to the pancreatic primary may also induce a "selection pressure" for the development of new genetic events remains unknown.

2. Tumor heterogeneity with distinct results in KRAS analysis at initial diagnosis (lymph node metastasis analyzed) and at progression (primary tumor analyzed):

There is increasing evidence for intratumoral heterogeneity in different types of cancer that could be determined by multiregion sequencing [19]. In non-small cell lung cancer it was shown that ALK rearrangements (that were previously thought to be mutually exclusive with activating EGFR and KRAS mutations) can be found together with EGFR mutations in rare cases [20]. Moreover, it was shown that spatially separated subclones of the same tumor harbor different oncogenic drivers [21]. If these observations are transferable to PDAC, this might explain the differences in KRAS mutational status observed in our patient reported here. However, the scarce currently available data comparing pancreatic primary tumors and corresponding metastases, showed the same KRAS mutational status in the primary tumor and each metastatic site examined, thus supporting the idea of a newly apparent KRAS mutation [22, 23].

3. Technical aspects of the discrepant KRAS sequencing results (see Fig. 2):

Potentially, the initial KRAS wildtype status detected in 2010 could be the effect of a false negative sequencing result. Both KRAS analyses in the tumor tissue of the patient reported here were performed 


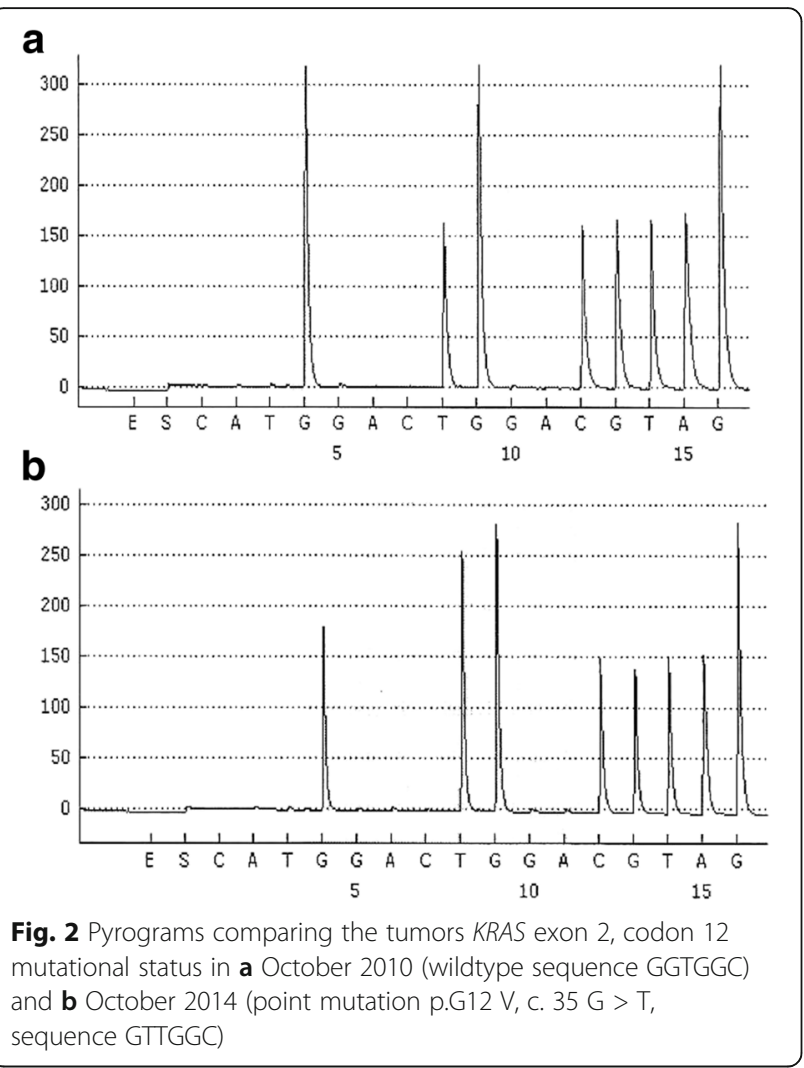

in the same specialized and certified laboratory for molecular pathology. For both analyses, formalin fixed paraffin embedded (FFPE) tumor tissue was microdissected under visual control using a microscope to reduce contamination by adjacent normal tissue. In both situations, sufficient tumor tissue was available: In 2010 a subtotally infiltrated lymph node metastasis, $22 \mathrm{~mm}$ in diameter, containing insignificant residual lymphatic tissue and in 2014 whole tumor resection tissue was used for analysis. Moreover, the pyrosequencing assay employed here is highly sensitive and requires only $10 \%$ of tumor DNA in the whole DNA extracted to reliably detect the KRAS mutational status [24]. Thus, a false negative sequencing result is a very unlikely event to explain the discrepancy in the present case.

\section{Conclusions}

KRAS mutational status may change during the course of disease in PDAC. Thus, in well-defined clinical scenarios (e. g. relapse after surgery in curative intent, disease progression during/after chemotherapy, unusual clinical course) a re-assessment of the KRAS status should be discussed, specifically within the setting of controlled clinical and translational trials. As KRAS is not yet established as a clinically relevant biomarker in
PDAC, future translational trials in pancreatic cancer that evaluate a broad range of novel biomarkers should, at least to our opinion, include a repeated biomarker assessment during the course of disease within their prospective study protocols. Novel promising techniques like liquid biopsy approaches may thereby help to overcome the limitations of obtaining tumor tissue safely in PDAC [25]. As it may be difficult to obtain sufficient tumor tissue in PDAC by percutaneous- or endosonography-guided biopsy techniques, a sampling error may occur specifically in the light of tumor heterogeneity. In that context, liquid biopsy techniques may also eventually help to overcome these limitations.

\section{Abbreviations}

5-FU: 5-fluorouracil; ALK: Anaplastic lymphoma kinase; CA19-9: Carbohydrate antigen 19-9; CDX2: Caudal type homeo-box transcription factor 2;

CK: Cytokeratin; CRC: Colorectal cancer; CT: Computed tomography; EGFR: Epidermal growth factor receptor; ERBB2: Human epidermal growth factor receptor 2; FFPE: Formalin fixed paraffin embedded; FOLFIRINOX: Folinic acid, 5-FU, irinotecan, oxaliplatin; Gy: Gray; KRAS: Kirsten rat sarcoma viral oncogene homologue; nab-paclitaxel: Nanoparticle albumin-bound paclitaxel; OS: Overall survival; PDAC: Pancreatic ductal adenocarcinoma; UICC: International union against cancer

\section{Acknowledgments}

We thank all the lab technicians at the Institute of Pathology for their excellent technical support.

\section{Funding}

SO is supported by grants from the Friedrich-Baur-Stiftung, Munich and the association for the promotion of research and science at the medical faculty LMU (wifomed), Munich.

\section{Availability of data and materials \\ Not applicable.}

\section{Authors' contributions}

SB, SO, AR, TK and AJ did the pathological investigations and the molecularpathological analyses of the reported case. MH, SK, DPM, VH and SB were the treating oncologists. JW was the surgeon who performed the pancreaticoduodenectomy. SB, SO, VH, AJ and SB designed the study, collected the clinical data and drafted the manuscript. All authors have read and approved the manuscript of this case report.

\section{Authors' information}

The authors are experienced pathologists, oncologists or surgeons involved in the multidisciplinary management of pancreatic cancer patients at the comprehensive cancer center of a large tertiary care university hospital.

\section{Competing interests}

The authors declare that they have no competing interests.

\section{Consent for publication}

The patient reported here was included in a single-center translational study protocol of the Ludwig-Maximilians-University of Munich, named "The Informative Patient" (Patient number 1303). Within that protocol, the patient gave written informed consent for data analysis and publication. Additionally, by signing the official consent form provided by BMC Cancer (http://resourcecms.springer.com/springer-cms/rest/v1/content/6621850/data/v1/ConsentForm-PDF), the patient gave written informed consent for publication of her data in form of this case report. Both a copy of the original informed consent for study participation, data analysis and publication (in German language only) as well as a copy of the original BMC Cancer consent to publish form are available for review through the editors of this journal. 


\section{Ethics approval and consent to participate}

The present translational study protocol was approved by the local ethics committee of the Ludwig-Maximilians-University of Munich (approval number 284-10).

\section{Author details}

'Institute of Pathology, Ludwig-Maximilians University of Munich, Munich, Germany. ${ }^{2}$ Department of Internal Medicine III and Comprehensive Cancer Center, Klinikum Grosshadern, Ludwig-Maximilians University of Munich, Marchioninistr. 15, 81377 Munich, Germany. ${ }^{3}$ Department of General, Visceral, Vascular and Transplantation Surgery, Klinikum Grosshadern, Ludwig-Maximilians-University of Munich, Munich, Germany. ${ }^{4}$ DKTK, German Cancer Consortium, German Cancer Research Center (DKFZ), Heidelberg, Germany.

Received: 10 March 2016 Accepted: 19 May 2017

Published online: 26 May 2017

\section{References}

1. Siegel RL, Miller KD, Jemal A. Cancer statistics, 2016. CA: A cancer journal for clinicians; 2015.

2. Eser $\mathrm{S}$, et al. Oncogenic KRAS signalling in pancreatic cancer. $\mathrm{Br} J$ Cancer. 2014; 111(5):817-22.

3. Burris HA 3rd, et al. Improvements in survival and clinical benefit with gemcitabine as first-line therapy for patients with advanced pancreas cancer: a randomized trial. J Clin Oncol. 1997;15(6):2403-13.

4. Heinemann $V$, et al. Randomized phase III trial of gemcitabine plus cisplatin compared with gemcitabine alone in advanced pancreatic cancer. J Clin Oncol. 2006:24(24):3946-52.

5. Heinemann $\vee$, Haas M, Boeck S. Systemic treatment of advanced pancreatic cancer. Cancer Treat Rev. 2012:38(7):843-53.

6. Goldstein, D., et al., nab-Paclitaxel plus gemcitabine for metastatic pancreatic cancer: long-term survival from a phase III trial. Journal of the National Cancer Institute, 2015. 107(2): p. dju413.

7. Shin SH, et al. Genetic alterations of K-ras, p53, c-erbB-2, and DPC4 in pancreatic ductal adenocarcinoma and their correlation with patient survival. Pancreas. 2013;42(2):216-22.

8. Sinn BV, et al. KRAS mutations in codon 12 or 13 are associated with worse prognosis in pancreatic ductal adenocarcinoma. Pancreas. 2014;43(4):578-83.

9. Miglio $U$, et al. KRAS mutational analysis in ductal adenocarcinoma of the pancreas and its clinical significance. Pathology-Research and Practice. 2014; 210(5):307-11.

10. Lee J, et al. Impact of epidermal growth factor receptor (EGFR) kinase mutations, EGFR gene amplifications, and KRAS mutations on survival of pancreatic adenocarcinoma. Cancer. 2007;109(8):1561-9.

11. Boeck $S$, et al. EGFR pathway biomarkers in erlotinib-treated patients with advanced pancreatic cancer: translational results from the randomised, crossover phase 3 trial AIO-PK0104. Br J Cancer. 2013:108(2):469-76.

12. Kruger $S$, et al. Translational research in pancreatic ductal adenocarcinoma: current evidence and future concepts. World J Gastroenterol: WJG. 2014; 20(31):10769.

13. Kim ST, et al. Impact of KRAS mutations on clinical outcomes in pancreatic cancer patients treated with first-line gemcitabine-based chemotherapy. Mol Cancer Ther. 2011;10(10):1993-9.

14. Boeck $S$, et al. KRAS mutation status is not predictive for objective response to anti-EGFR treatment with erlotinib in patients with advanced pancreatic cancer. J Gastroenterol. 2013;48(4):544-8.

15. Allegra, C.J., et al., Extended RAS gene mutation testing in metastatic colorectal carcinoma to predict response to anti-epidermal growth factor receptor monoclonal antibody therapy: American Society of Clinical Oncology provisional clinical opinion update 2015. Journal of clinical Oncology, 2015: p. JCO 2015.63. 9674

16. Wolff $A C$, et al. Recommendations for human epidermal growth facto receptor 2 testing in breast cancer: American Society of Clinical Oncology/ College of American Pathologists clinical practice guideline update. Arch Pathol Lab Med. 2013;138(2):241-56.

17. Misale $\mathrm{S}$, et al. Emergence of KRAS mutations and acquired resistance to anti-EGFR therapy in colorectal cancer. Nature. 2012;486(7404):532-6.

18. Diaz LA Jr, et al. The molecular evolution of acquired resistance to targeted EGFR blockade in colorectal cancers. Nature. 2012;486(7404):537-40.
19. Gerlinger $\mathrm{M}$, et al. Intratumor heterogeneity and branched evolution revealed by multiregion sequencing. N Engl J Med. 2012;366(10):883-92.

20. Birkbak NJ, Hiley CT, Swanton C. Evolutionary precision medicine: a role for repeat epidermal growth factor receptor analysis in ALK-rearranged lung adenocarcinoma? J Clin Oncol. 2015;33(32):3681-3.

21. Cai, W., et al., Intratumoral heterogeneity of ALK-rearranged and ALK/EGFR coaltered lung adenocarcinoma. Journal of Clinical Oncology, 2015: p. JCO. 2014.58. 8293.

22. Embuscado EE, et al. Immortalizing the complexity of cancer metastasis: genetic features of lethal metastatic pancreatic cancer obtained from rapid autopsy. Cancer biology \& therapy. 2005;4(5):548-54.

23. Yachida $\mathrm{S}$, et al. Distant metastasis occurs late during the genetic evolution of pancreatic cancer. Nature. 2010:467(7319):1114-7.

24. Ogino $S$, et al. Sensitive sequencing method for KRAS mutation detecting by pyrosequencing. The Journal of Molecular Diagnostics. 2005;7(3):413-21.

25. Kinugasa $\mathrm{H}$, et al. Detection of K-ras gene mutation by liquid biopsy in patients with pancreatic cancer. Cancer. 2015;121(13):2271-80.

\section{Submit your next manuscript to BioMed Central and we will help you at every step:}

- We accept pre-submission inquiries

- Our selector tool helps you to find the most relevant journal

- We provide round the clock customer support

- Convenient online submission

- Thorough peer review

- Inclusion in PubMed and all major indexing services

- Maximum visibility for your research

Submit your manuscript at www.biomedcentral.com/submit
C) BioMed Central 